\title{
Optimization of ICT Common Wealth Planning and Sharing based on Organic Economic Ecology and Theory of Knowledge Value Transformation on case of open collaboration Framework
}

\author{
Johannes K. Chiang \\ Department of Management Information Systems \\ Federated Cloud Big Data and Service Innovation Research Centre \\ National Chengchi University \\ No.64, Sec.2, ZhiNan Road, Wenshan District, Taipei City 11605,Taiwan (R.O.C) \\ E-mail: jkchiang@nccu.edu.tw
}

\begin{abstract}
Since the advent of open science, ICT resources become the common wealth. For the common wealth planning and sharing, the author has carried out HyQVIS (Hyper Quality and Value of ICT Systematics). Then, the author further carried out the Theory of the Knowledge Value Transformation. According to the theory, the task of resource planning and sharing is to optimize the behaviour of the organic ecosystem after appropriate laws. This research applies Pareto Optimality as the optimization method for planning and sharing the resources and further use Pareto Frontier to improve the efficiency. In order to realize Pareto Frontier with scientific base, this research adopts ISO 25000 to define the metrological metrics for the features derived from Pareto Frontier. Based on this theoretical baseline, this research conducts a case study of open collaboration framework with respect to API and Platform Economy. This research applies further the Shapley value with consideration of inclusive economy to enhance the cooperative competition strategy. For this purpose, comprehensive factors influencing the Organic Ecosystem are considered. On top of these consideration, different organization structures of the ecosystems are constructed. The complexity, including efficiency and risk, availability and value are analysed. The result shows, "open" is the best consensus for optimization of the ecosystem, and the federated cloud is most efficient as well as less of complexity and costs, less of risks. Federated Cloud becomes the next edge of the Infrastructure of collaboration framework in the API and Platform Economy.
\end{abstract}

International Symposium on Grids \& Clouds 2020, ISGC2021

22-26 March, 2021

Academia Sinica, Taipei, Taiwan (online)

*Johannes K. Chiang

(C) Copyright owned by the author(s) under the terms of the Creative Commons

Attribution-NonCommercial-NoDe

rivatives 4.0 International License (CC BY-NC-ND 4.0).

https://pos.sissa.it/ 
Optimization of ICT Common Wealth Planning and Sharing based on Organic Economic Ecology and Theory of Knowledge Value Transformation

Johannes K. Chiang

\section{Introduction}

\subsection{Organic Ecology - HyQVIS}

The author has carried out the paradigm of Organic Ecology in 1994 [1]. Besides the Ecology from Biology, the author emphasizes synergetic [2] and introduced Chinese Taoist's world of Yin (Female, Earth) as well as Yang (Male, Haven) and inter-promoting as well as interinhibition relation of the elements the world [3] into the Organic Ecology. Inter-inhibition is often interpreted as competition in English and inter-promoting highlights cooperation [1]. Originally, ecology is a branch of biology concerning the distribution and abundance of organisms [6] but later the western academicians consider mostly the biologic evolution of Darwin which emphasizes the mutation and elitism but ignore often the organism. Therefore, the author brings the aspect of Chinese Taoist's Transformation and Recycling of the domains of livings, which was discovered at first by the British historian Toynbee in the western world [7], and the external projection, i.e. learning and collaboration by the organic agent with counterparts in the ecosystem [1]. As a result, the HyPloy (Hyper Ploy) becomes a fusion function of External Excitation, Intrinsic Experience and Intention, and Extrinsic Projection to generate the Response that can be feedback to Intrinsic Experience for evolutional improvement or trigger the next cycle of action, as shown in Fig. 1. The term "Hyper" is used to emphasize the marriage of female and male of Taoism as well as crossover of biological evolution.

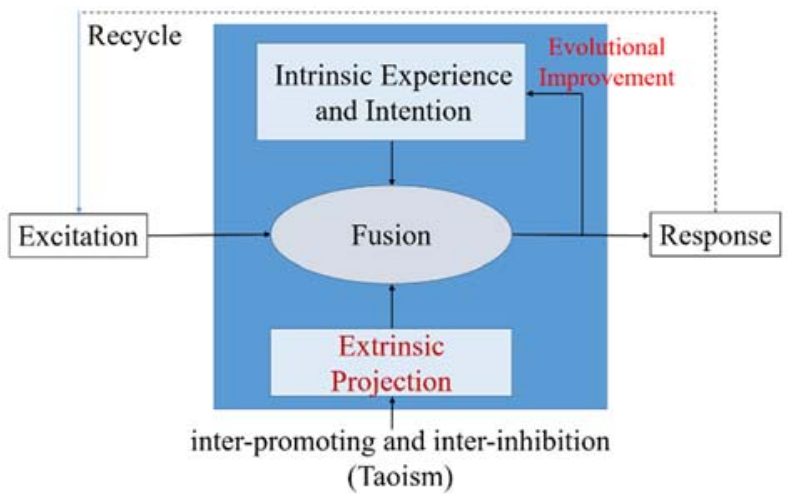

Fig. . HyPloy Organic Ecology Model

With further consideration of ICT Ecosystem based on Shannon's Information Theory [8], HyPloy is used to construct the open system encompassing activities on three levels, i.e. communication, cooperation and coordination that implies optimization, on top of information network as the repository, as shown in Fig. 2. This model highlights the interoperation of the organic agents to overcome the problems of the isolated island solution, viz. close-world internal strategy, unreliable information exchange, high quality cost. It is worth to mention that focusing on internal strategy is still the obstacle for developing open ecosystems. Pfeifer and Chiang developed then the Hypertext Quality Information Systematics (HyQIS) which is the core of Kaizen (Continuous Improvement of Hanji) for Total Quality Management (TQM) and was realized within the EU FP3-ESPRIT Project 8733 [9] which also addressed the concept of Quality of Service (QoS). However, at that time, HyQIS only considered the quality. Since middle 1990s, the economic drive has shifted from "managing scarcity" to "producing abundance"[10], therefore the author conducted further a research into concept of value regarding Roger's Innovation Diffusion Theory [11] where the author introduced Confucius pragmatism and altruism [5] for 
Optimization of ICT Common Wealth Planning and Sharing based on Organic Economic Ecology and Theory of Knowledge Value Transformation

Johannes K. Chiang

value aspect and extended the HyQIS to HyQVIS (Hyper Quality and Value Information Systematics) [4][5], And therefore the value is comparative in HyQVIS. Since long, the western economists usually emphasize only competition but ignore cooperation. Hence, the author carried out the competition-cooperation strategy [4] [5].

Isolated Island Solutions, Sequential Works

Marketing R\&D Production QA

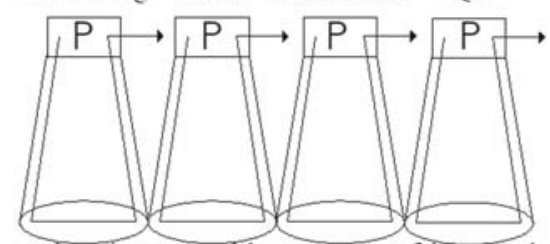

process implemented by means of internal strategy

unreliable information transfer

long process time

high quality cost

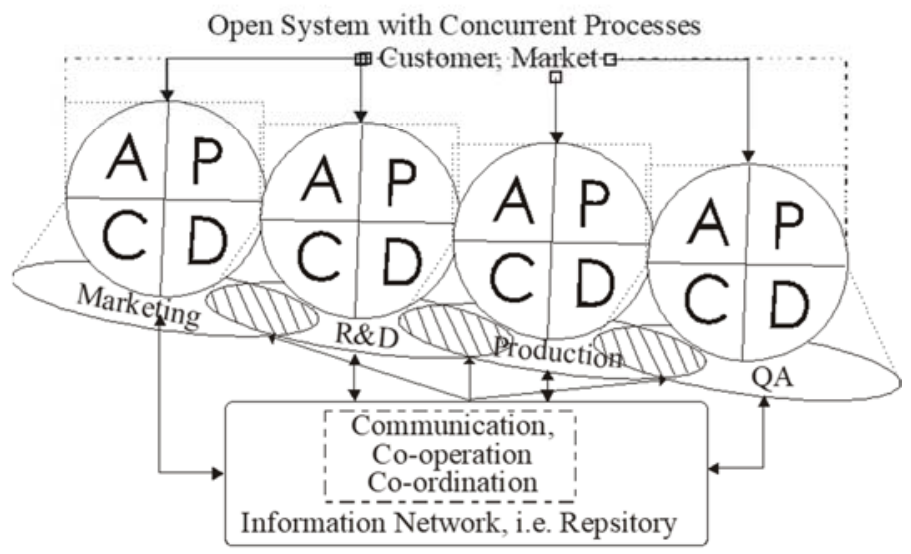

to make development in-line with market situations

to dismish information lost

to make the process time shorter

to reduce quality cost

Fig. 2 Organic Ecosystem

\subsection{Theory of Knowledge Value Transformation}

Based on HyQVIS, the author proposes the Knowledge Value Transformation Theory. In order to conduct value based strategy, the feature and value has to be identified through a planning phase [1]. Assume the behavior of individual organic agents in an organization can be separated into two parts: the common part and the particular part. The common part is the indifference features within the organization that are maintained by the similar supported activities of the HyQVIS ecosystem. The particular part is the distinct operation structure according to individual's domain to supports its unique status. Now the comparable feature domain could be seen as a unified feature space preserving the landscape of the ecosystem and detail weights of the constituents. With the organization's features, we can prepare the transform function for the relations between the common features, individual features, and the comparable feature space to generate the comparative value of each individual agent. The function transforms the commonality, whereas maintains the source generality. The optimization can be given by:

$$
\arg \min _{A, U} \sum_{c \in S, T} \sum_{i=1}^{j} L\left[y_{c_{i}},\left(A_{c}, U^{T} \overrightarrow{x_{c_{i}}}\right)\right]+\lambda \mathcal{L}
$$


Optimization of ICT Common Wealth Planning and Sharing based on Organic Economic Ecology and Theory of Knowledge Value Transformation

Johannes K. Chiang

Starting from the center part of the individual $\mathrm{i}$ with its common quality feature vector $\left(\mathrm{x}_{c i}\right)$, we would like the part of sources $\mathrm{S}$ maps to common quality feature space of Target $\mathrm{T}$ by an organic function, which contains the mapping function $\mathrm{Us} \rightarrow \mathrm{t}$ and parameter matrix $\mathrm{A}$. We need to run this loop until all $\mathrm{j}$ individual $(\mathrm{i} \in \mathrm{j}$ ) complete the mapping and create the common feature space $\mathrm{Y} c$.

The objective is to find the argument sets that can minimize the cost by optimizing A and U. The organic function is also the affine translation that combines the vector space of common feature domain and individual feature onto the new space of comparable feature domain.

With the features of the ecosystem, we can prepare the transform function for the relations between the common features, individual features and the comparable feature space to generate the comparative value of each individual organic agent.

The optimization should obey a certain law or de factor consensus that reflects a moral spirit. The overall systematics is a Transformer from knowledge domain on the semantic level to comparative value domain on the pragmatic level in terms of Automata.

\section{Economic Optimization Method}

Pareto Optimality comes form the economics and emphasizes impovement, that is the key point of HyQVIS. This reserach adopts it for optimization as needed by the theory of Knowledge Value transformation.

\subsection{Pareto Optimality}

The notion of Pareto Optimality has been applied to the selection of alternatives in engineering and biology, besides economics [12]. Thus, Pareto Optimality is a sit uation where no individual or preference criterion can be better off without making at least one individual or preference criterion worse off or without any loss thereof. The concept is named after Vilfredo Pareto, who used the concept in his studies of economic efficiency and income distribution. The following three concepts are closely related:

- Given an initial situation, a Pareto improvement is a new situation where some agents will gain, and no agents will lose.

- A situation is called Pareto dominated if there exists a possible Pareto improvement.

- A situation is called Pareto optimal or Pareto efficient if no change could lead to improved satisfaction for some agent without some other agent losing or if there's no scope for further Pareto improvement.

\subsection{Pareto Frontier}

The Pareto frontier is the set of all Pareto efficient allocations. It also is variously kn own as the Pareto front or Pareto set. Given a set of choices and a way of valuing them, the frontier or Pareto set or Pareto front is the set of choices that are Pareto efficient. By restricting attention to the set of choices that are Pareto-efficient, a designer can make tradeoffs within this set, rather than considering the full range of every parameter.

Pareto efficiency is the state in which there is no room for Pareto improvements, which are regarded as the lower boundary of the ideal state in an economy. If an innovation has surpassed the previous efficiency postulation or a new dimension is defined as the new target, the Pareto frontier boundary can be considered with respect to Inclusive Economy as following:

- Aviaibility of sevices (Access) 
Optimization of ICT Common Wealth Planning and Sharing based on Organic Economic Ecology and Theory of Knowledge Value Transformation

- Application content and efficiency (Usage)

- Goodness of fit and Intelligibility of services(Quality)

These three dimensions can be improved throught open ecosystem.

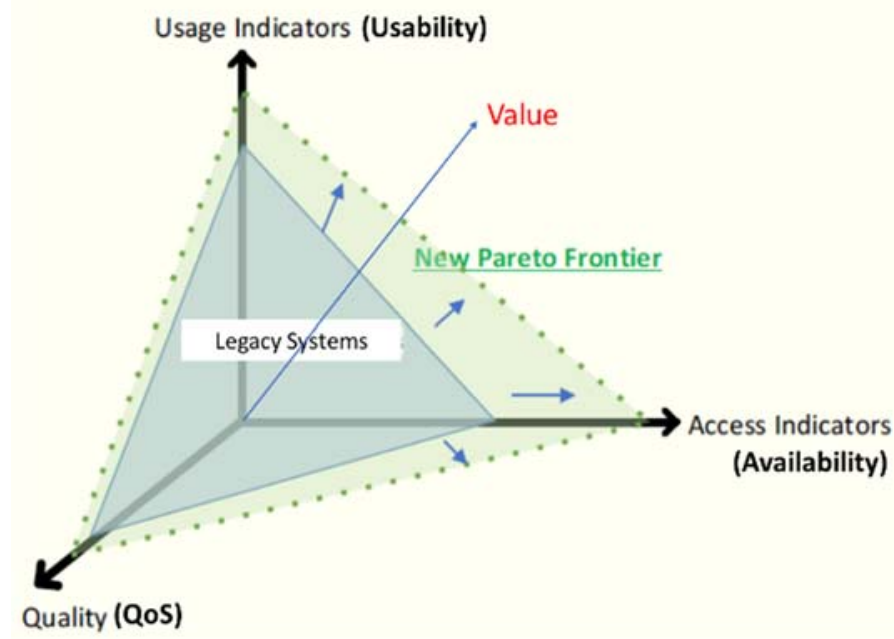

Fig. 3 Pareto Frontier

\subsection{Metrics for Pareto Frontier - ISO 25000}

For the Pareto Frontier, there should be a way of valuing the given a set of choices, this rsearch adpots ISO 25000, also known as SQuaRE (System and Software Quality Requirements and Evaluation) [13] as the methrology metrics to bulid the scientific foundation for the features of ecosysyems. The festues of Pareto Frontier can be described according to ISO 8402 and ISO 9000 [1] as following:

1. Quality $\sim$ QoS: Degree to which the response and processing times and throughput rates of a resource or a system, when performing its functions, meet requirements. [14]

- Throughput

- Efficiency

- Latency

2. Measure of Availability: Degree to which the amounts and the maximum limits of a resource or system parameter meet requirements.

- Amount of the resources

- Uptime: the measure of time a given service is up and available to be used by the appropriate end users.

- Downtime: not available to the end users

3. Measure of Usability: Degree to which types of resources used by a system or user, when performing its functions, meet requirements.[15]

- Functional completeness - degree to which the set of functions covers all the specified tasks and user objectives.

- Functional correctness - degree to which a product or system provides the correct results with the needed degree of precision.

- Functional appropriateness - degree to which the functions facilitate the accomplishme nt of specified tasks and objectives including Easy to Use and Usefulness (TAM: 
Optimization of ICT Common Wealth Planning and Sharing based on Organic Economic Ecology and Theory of Knowledge Value Transformation

Johannes K. Chiang

Technology Acceptance Model), and Compatibility, viz. co-existence and interoperability.

\subsection{Value for HyQVIS}

Along with ICT-driven paradigm sift of conomy, Open API and Platform Economy [16] become the edge of the development of ecosystems. For this, this this research defines the metrics for the value w.r.t. HyQVIS. The organic ecosystem framwork consists of the planner, provider and user. For the three kinds of role of participants in the econsystem, the values of planner, provider and user can be described as following:

1. Real Value, usually measured by money

2. Comparative Value:

- Planner Value includes the Planned Outcome, Planned Benefits (Incomes) \& Costs and Product/Service. This has to be fixed in the planning phase, including the a availabie resources(Availability), the expected incomes and costs and product of the planning phase. The optimization starts form this phase.

- Expected value comprises Causality, Expected Outcome and Expected Atributes. The optimization of shraing starts from the this phase.

- Perceived Value incluses Final Outcome/Service, benefits \& costs and meaningful attributes. Since it is "perceived", it is compartive and often needs restropective reseacrh into user experiences for the checking activity. The results can triger the improvememt of the following lifecycle, so it can be used for HyQVIS.

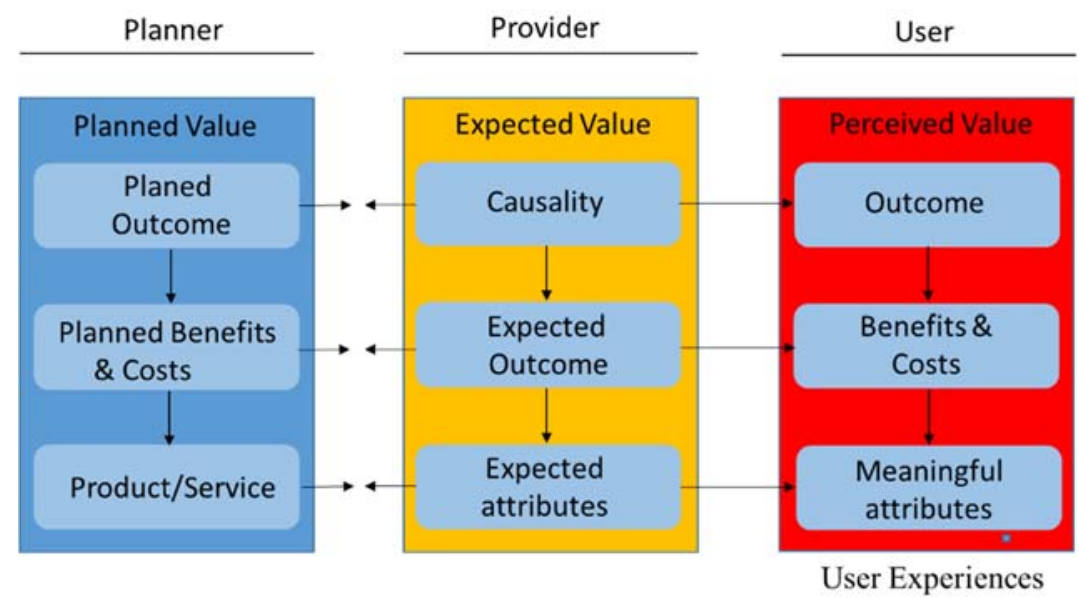

Fig. 4 Extended Value for API and Platform Economy

\section{Case study of Open Collaboration Framework centric to Federated Cloud}

\subsection{Open Collaboration Framework [17]}

Disaster Mitigation Competence Centre (DMCC) has started to build up an open collaboration framework. Simulation portals is developed from thoroughly investigated case studies. The whole hazardous event is able to be reproducible from the collaboration framework. The simulation facility will be continuously enhanced from more case studies. Regional distributed cloud infrastructure is used to support the workflow of simulations, data management and sharing, and col-laborations. Through the open collaboration framework, partners are able to 
Optimization of ICT Common Wealth Planning and Sharing based on Organic Economic Ecology and Theory of Knowledge Value Transformation

Johannes K. Chiang

access seamlessly to the knowledge from case studies, to conduct new case studies by making use of the simulation facilities, and to reuse or repurposing all resources.

A Knowledge Base is used to share all the materials and resources of DMCC case studies in an organized way. It is implemented over the DMCC Open Collaboration Platform to support hazard risk analysis by making use of DMCC services and resources. The knowledge base will also provide the collective intelligence environment for partners to conduct new case studies or reproduce and reinvestigate existing cases.

The open collaboration framework consists of the core multi-disciplinary taskforce and the technical platform. The technical platform contains online services for simulation, data and information management in collaboration with both EGI and Asia partners. The taskforce covers scientific group, technical group, e-Infrastructure group and user support group. The primary functions of taskforce are to identify case studies; collect observation data and supporting materials; develop simulation models; validate the models based on historical observation scenarios; integrate the model and data with e-infrastructures; conduct performance tuning; deploy the scenarios and tools into online portals.

Knowledge Base is used to share all the materials and resources from case studies in an organized way. It is implemented over the designed Open Collaboration Platform to support the utilization of services and resources and provide the collective intelligence environment for partners to conduct new case studies or reproduce and reinvestigate existing cases. The Open Collaboration Platform maintains the data federation that provides flexibility to check up the basic information of events from those reliable regional and global information.

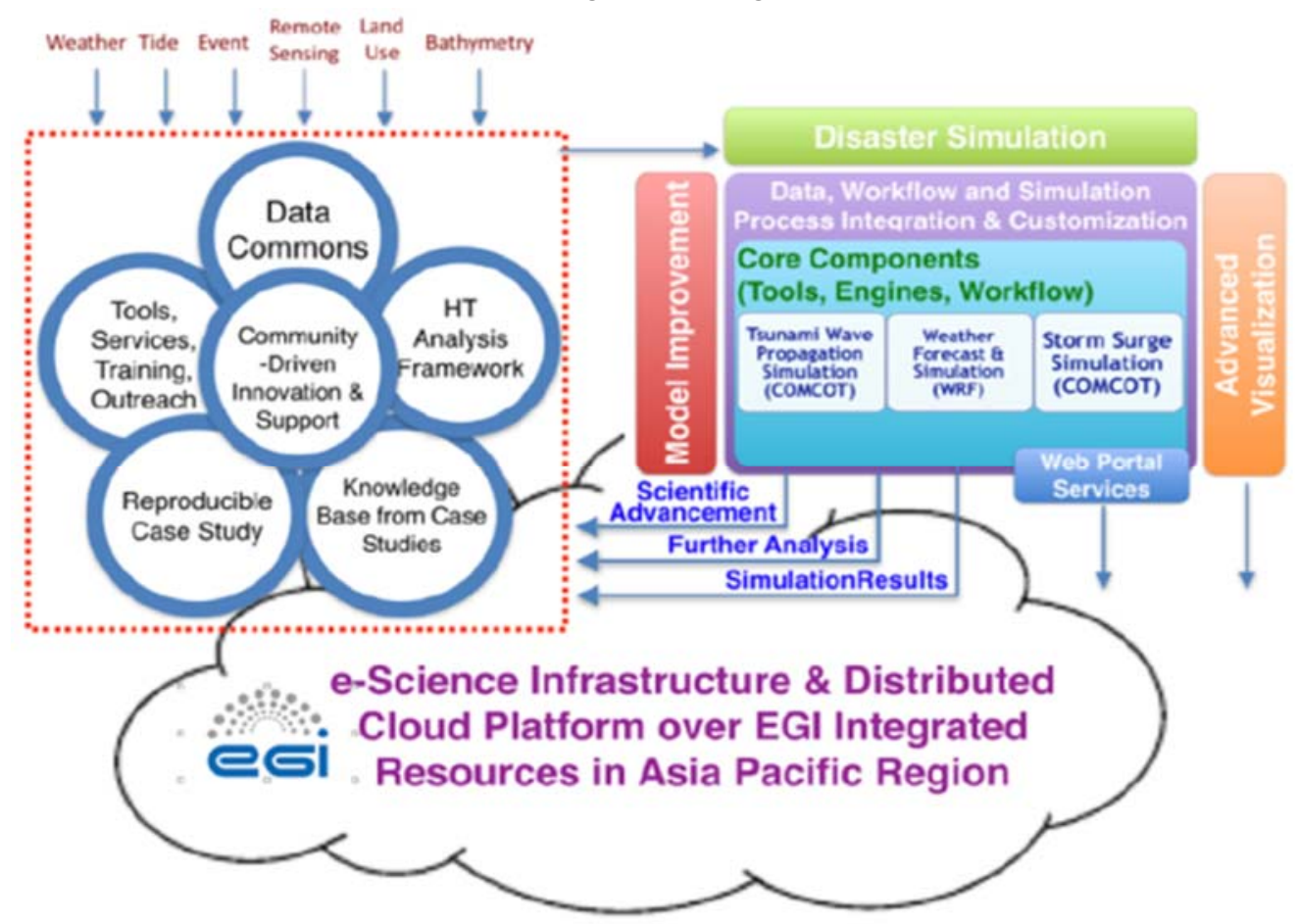

Fig. 5 Moving Towards Open Science Platform from DMCC Collaboration Framework

\subsection{Federated Cloud [18]}

A federation is a group of computing or network providers agreeing upon standards of operation in a collective fashion. The term may be used when describing the inter-operation of 
Optimization of ICT Common Wealth Planning and Sharing based on Organic Economic Ecology and Theory of Knowledge Value Transformation

Johannes K. Chiang

two distinct, formally disconnected, telecommunications networks that may have different internal structures. The term federated cloud refers to facilitating the interconnection of two or more geographically separate computing clouds. The term may also be used when groups attempt to delegate collective authority of development to prevent fragmentation.

Plenty of institution today are in different stages of cloud adoption. New market trends and evolving business models are renovating the entire computing industry to focus more on a variety of cloud services from different providers that bring value to customers. Those institutions are endeavoring to adopt the hybrid cloud ecosystem model, which encompasses administration of their traditional workloads as well as the workloads that are distributed across various clouds. The increasing prominence of hybrid cloud environments in digital transformation is leading institutions to accentuate on innovative platforms, partnerships, agreements, regulations, designs, and federations.

Cloud federation provides a platform for fabricating a hybrid cloud ecosystem in a seamless manner with no vendor locks, giving customers a magnitude of players to choose from based on their budgets and investment plans. It also encourages users to switch from one platform to another with minimum risk and costs.

\subsection{Platform Economy [16]}

In the Platform Economy, a platform is a economic service model that creates value by facilitating exchanges between two or more interdependent groups, usually users and providers. This term is used by analysts to describe the competitive nature of digital innovation. In order to make these exchanges happen, platforms harness and create large, scalable networks of users and resources that can be accessed on demand.

Platform economy is the tendency for computing to increasingly move towards and favor digital platform service models. Platforms are underlying computer systems that can host services that allow users, entrepreneurs, institutions and the general public to connect, share resources or promote their services in terms of collaboration. And, it results in so-called digital transformation.

\subsection{API Economy [16]}

In API Economy, institution utilizes resources efficiently and quickly to create added value for own users. These resources can be for example data or function provided by other organizations. Building blocks utilized are own APIs and open APIs provided by other organizations (free or commercial) in addition to developer communities. These enable quicker adaptability to unpredictable and faster changing user needs. Defining characteristics of API Economy are competing for popularity among application developers and considering them as primary users. In brief, services are offered from organizations to developers.

APIs enable interaction with platform economy operators. One's service model may encounter a "forced opening" of APIs at any time - see it as an opportunity. The API economy is not just part of platform economy, but APIs can be used for increasing internal productivity or for offering different service models. Shared Economy

Sharing Economy is the idea that users would prefer to share resources/services, esp. Open Data, and thus information and services become common wealth. The platform economy facilitates this as an institution can develop a digital economic platform that users can log onto to 
Optimization of ICT Common Wealth Planning and Sharing based on Organic Economic Ecology and Theory of Knowledge Value Transformation

gain access to resources/services through a subscription. The platform service providers earn incomes from externality of economics.

\section{Research Design}

HyQVIS emphazes the improvement throught cooperative competition strategy. The optimization for the panning and sharing of resources is in the fact a federation gaming. This research takes the game theory into account.

\subsection{Ideal state equally according to Cooperative Competition Strategy of HyQVIS}

The Shapley value [19] is a solution concept for cooperative game theory. It's the vital few of western economic strategy that considers cooperation as HyQVIS does. To each cooperative game it assigns a unique distribution (among the players) of a total surplus generated by the coalition of all players. The Shapley value is characterized by a collection of desirable properties. The Shapley value for the ideal state of equally share benefit from the open collaboration Framework. Let the cooperation S has a fair way payoff so the members should receive benefits in proportional to their marginal contributions. The payoffs for each player $i$ based on the marginal contribution can be divided by the value equation as:

$$
\phi i(v)=1 / \mathrm{N} ! \Sigma \mathrm{S} \subseteq \mathrm{N} \backslash\{\mathrm{i}\}|\mathrm{S}| !(|\mathrm{N}|-|\mathrm{S}|-1) ![\mathrm{v}(\mathrm{S} \cup\{\mathrm{i}\})-\mathrm{v}(\mathrm{S})]
$$

The result is equal in distribution, but dis-incentivizes the motivation to join the cooperation. For the dominant participant $b$,

$$
\phi b=2.5<v(a, b) \times 2 /(1+2)=2.67
$$

The anticipation of such distribution might result in an unstable outcome that forces $b$ to leave the grand federation and form another smaller federation for redistribution.

\subsection{Feasible state stable core}

Obviously, federation needs a stable equilibrium as HyQVIS emphasizes. It needs to be founded on the selfish property to redesign the mechanism by the core set in open game. The core condition is an un-dominated imputation set that obtains the stable payoff of Pareto efficiency in which no agent would suffer. Core condition is an un-dominated imputation set that obtains the stable payoff of Pareto efficiency in which no agent would suffer from the new criterion of state modification or resource reallocation.

\subsection{Dominant Participants}

Let's examine the influences from dominant participants in an open game. Generally, there are a variety of organic agents in different scales of organization. Among them, the largest authorities play the central role in the success of open framework.

In practice, major organic agents often come from different associations to assist the government authority in implementing policies. Normally, all major organic agents are required to join the collaboration, which often reports to the authority for self-discipline conventions. These records are deemed part of the self-regulations for the members, even though the compliance of these decisions is not mandatory in law.

If we want to manage with both stability and fairness in such game, we need the dominant participants make the concession. The Shapley Value $\phi$ is then calculated under condition of:

$$
\phi e(v)=2 / 6 * 1+1 / 6 * 1+1 / 6 * 1=2 / 3 . \phi p(v)=1 / 6 * 1=1 / 6=\phi q(v) .
$$


Optimization of ICT Common Wealth Planning and Sharing based on Organic Economic Ecology and Theory of Knowledge Value Transformation

Johannes K. Chiang

This solution suggests giving $e$ more and sharing the rest equally to $p$ and $q$ based on their marginal contribution. One important implication of this is that the collective action in the collaboration must generate synergy as HyQVIS intends so it is no less than their union set. The above analysis has clearly indicated how necessary it is to ask the dominant agents $\mathrm{s}$ to join an open collaboration and the imperative bindings in it.

\subsection{Cooperation of Major Service Provider (MSP) and Third Party Partners(TPP)}

The collaboration between MSP and TPPs is something different from that of only authority agents. MSP and TPPs have different genes, but in open framework they need to unite and take actions to make the relevant unions work according to their expectations. In such cooperation, conflicts of interest are likely to occur and affect the group's decisions and actions. From the perspective of economic position, MSP are generally much larger than TPPs.

They inherit the irreplaceable legitimate power from the administration, and informational power from the data flows and insights of financial customers. HyQVIS emphasizes cooperation and Altruism. On designing the effective interaction mode between MSP and TPPs, we can analyze their payoff as:

Cooperation: MSP and TPPs cooperate with each other to discuss the collaborative framework. Both parties treat the other as a partner to meet same goals. The benefit is on both sides and equal flowing to each others as $M S P \leftrightarrow T P P$.

- Altruism: The MSP at its own cost builds a compatible environment for the business cooperation with the TPP and supports the development of the mutually shared infrastructure. The selected TPP counts heavily on the relationship with this MSP. The resource flow is $M S P \rightarrow T P P$.

- Spite: MSPs and TPPs treat each other as competitors in a zero-sum game, each developing one's own protocol to try to dominate the market without cooperative consensus. No one gets direct incomes from the other side.

- Selfishness: The TPP follows the existing protocol and business model of the MSP and customizes a specific environment for every MSP. The MSP receives benefits unilaterally from the TPP. The resource flow is $M S P \leftarrow T P P$.

Noticeably, altruism helps the emergence of the cooperation due to reciprocation. Customer data in the collaborative ecosystem could appear in different forms.

\begin{tabular}{|c|c|c|}
\hline & \multicolumn{2}{|c|}{ TPP } \\
\hline \multirow{4}{*}{ MSP } & $\begin{array}{c}\text { Altruism } \\
(-,+)\end{array}$ & $\begin{array}{c}\text { Spite } \\
(-,-)\end{array}$ \\
\cline { 2 - 3 } & $\begin{array}{c}\text { Cooperation } \\
(+,+)\end{array}$ & $\begin{array}{c}\text { Selfishness } \\
(+,-)\end{array}$ \\
\hline
\end{tabular}

Fig. 6 Payoff of Collaboration of MSP and TPP

If the MSPs all choose not to contribute first, then the open ecosystem development would be slow, and the authority would need to interfere to elevate the bargaining position of TPPs. In 
Optimization of ICT Common Wealth Planning and Sharing based on Organic Economic Ecology and Theory of Knowledge Value Transformation

Johannes K. Chiang

an equal status situation, cooperation is more likely to happen. This fact explains why the MSPs should be actively taking steps to build a friendly environment of open framework and assist TPPs to get on track quickly. This also explicates what the authority could do to help to facilitate cooperation among them.

\subsection{Hidden conflicts of Self-regulation and social welfare}

Building an open ecosystem based on the voluntarism and self-regulation principles are one of the widely adopted approaches among implemented economies. It is natural, because the management scope of the authority is only limited to authority institutions. With such arrangements, the authority is able to impose severe requirements on authority institutions and ask them to regulate the cooperation with the TPPs so as to extend the management to the ecosystem. From the perspective of responsible innovation and alleviation of impacts on society, this is also the quickest visible way to catch up with the open framework trend.

To engage in services, TPPs are fumbling for cooperation with MSPs and adaptation to the regulations. It is not possible for them to anticipate the risks of operations and the regulation requirements they need to meet. Therefore, it would be impractical to impose the same level of regulation that MSPs face onto TPPs. Compared to MSPs, the quickly evolving market is likely to incur more frequent replacements of TPPs. To prevent impairment of public confidence on the ecosystem, it would be better to set multiple light firewalls according to risks potentials. We should not resort to punishment to improve the quality of the open ecosystem, and neither should we expect the inspector to enforce that. To manage the behavior of TPPs in open framework, a flexible setting along an achievable learning curve could be more advantageous to the entire ecosystem. A quick responding and time to market supervisory vehicle with customized process of tutoring and counseling facilities should be devised.

Given the situation that minor agents decide the social welfare of the majority, such as the situation in which the authority decides on the development of open framework and economic inclusion, under conditions of agent preferences being possibly ordered in connected and transitive relations and their preferences are respected (Pareto efficiency).

\subsection{API security}

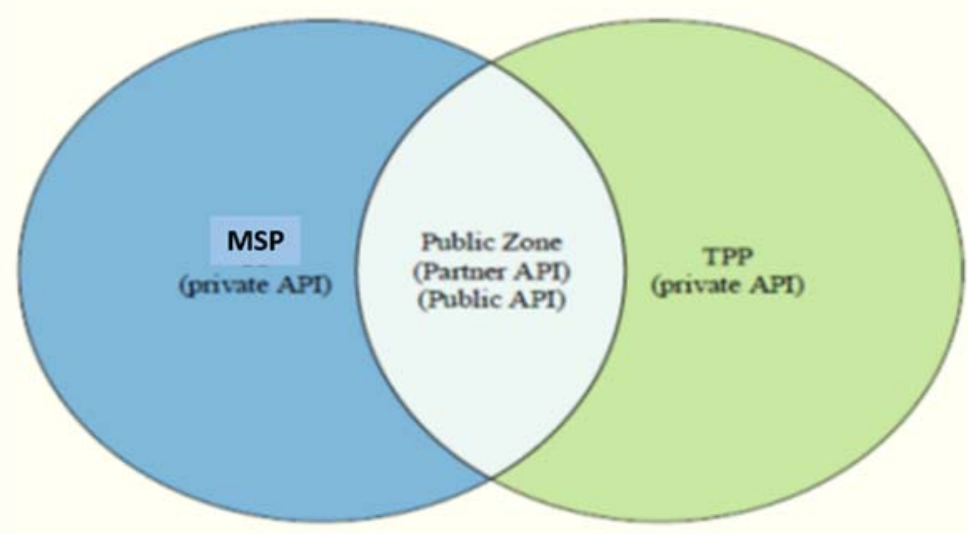

Fig. 7 Types of APIs in Open Collaboration Framework

- Private API: Internal procedure calls. 
Optimization of ICT Common Wealth Planning and Sharing based on Organic Economic Ecology and Theory of Knowledge Value Transformation

- Partner API: Information access between partners.

- Open API: open data access to public

API security depends the right setting but not the safety environment. Service has been classified to critical facility and the risk management jobs should be taken seriously in system level. Open ecosystem related management tasks are broad. Various API standards may bring in abundant regulatory effort. Standards may be outdated, have efficiency issues, or be incompatible with new protocols.

Therefore, standard unification and management is a continuous task. Besides, modern system vulnerability could be unknown, newly found, or neglected to repair, which could become breaches of invasion for open ecosystem. The strengthening of engineering fortification is also an uninterrupted work. Further, different software maturity levels of participants also bring certain challenges to management. Since APIs are developed by the participants (and their subcontractors) to connect the loosely coupled functions and form the open services, software development capabilities above a certain level will be necessary for subsequent testing and verification.

\subsection{Secure data exchange and the selection of protocol}

Global innovators, who focus on opportunities, normally prefer the lighter, elastic RESTful architecture to design APIs. To cope with flexibility and efficiency, the open framework requires to consider the connection of close protocol and the open specification, the intermediary DMZ (Demilitarized Zone)/Perimeter network and the throughput of them. This issue is not an easy task as the legacy system is the architecture based on heavier protocol. In order to support innovative TPPs, it needs to develop compatible RESTful service for the new comers and the coming IoT transactions. The layered network structure is a widely adopted hierarchy. Sensitive data shall be stored in SZ(System Zoom) only. SZ accepts the permitted connections only. Internet services for outside parties can only be carried out through DMZ. The Open Zone(OZ) only communicates with DMZ, and the DMZ connects SZ by way of reverse proxy. Indeed, this layer structure is the prevailing mechanism applicable to open API now.

The universality of API is an important principle in mind. It is difficult for participants outside existing systems to attend the closed proprietary. The open source solution which is largely adopted by TPP and maintained by open community is the mainstream in software development that needs to respect. So applies to cloud computing, container deployment, git commitment and modularization.

The compatibility is another important factor to consider. Some service providers have cooperated with TPPs prior to open framework. It is imperative to accommodate them in the open framework to avoid grey areas. The interim arrangement should be provided to help the convergence.

Once the API specification is standardized, it should be a mandatory, not voluntary protocol to follow and to synchronize, including data dictionary, version control, and authentication, authorization, and encryption requirements.

\section{Analysis and Results}

According to the aforemensioned influencing factors, this study constructed the ecosystems with different organization structures as following: 
1. Mode 1: Centralized regime: There is a designated and centralized organic agent serving as the information exchange platform to provide all the open API service. With a competent centralized hub, all participants should follow its requirements to enroll and apply open API contents and declare the progress of every version of API.

2. Mode 2: Multiple Centralized Organic Agents with unified rules. It is an ecosystem where no single DMZ exists. The implementation entity in this mode will not get involved in the practical infrastructure. Then, it could work with a regulated protocol. A unified protocol refers to that there is a standardized technical requirement and specification, and the protocol is compulsory to follow. With a regulated protocol.

3. Mode 3: Multiple Centralized Organic Agents without unified rules: It functions without a regulated protocol. Once there is no unified portal for the API communications, service providers have to build their own DMZs in order for their own services.

4. Mode 4: Partially Centralized Organic Agent: There is a nominated implementation entity, but it is only referential or performs part of the functions required for open framework. It happens if implementation entity is assigned only partial functions or responsibilities of open framework, such like it only demonstrates the use cases of shareable account information, or simply provides referential protocols for partner APIs. It is not formally entitled to maintain the order of the open ecosystem, so none of its announcement is compulsorily to comply with. It might build up a referential DMZ, but not all open transactions pass through this DMZ. It has a demonstrated unified standard, while this standard is for reference only. Under this circumstance, it would become just one of the open API platform providers except that it is a node with certain default edges

5. Mode 5: Decentralized Autonomous agents: In this mode, MSP or TPP tentatively build up more services that connect each other. Since there is no systematical picture, the API connection is limited to selected applications with specific partners. Open framework in this mode is equivalent to application innovation at discretion.

\subsection{Analysis of the complexity}

Fig. 8 shows the result of the analysis of the complexity of the five modes.

\begin{tabular}{|l|l|l|}
\hline Mode & \multicolumn{1}{|c|}{ Time Complexity } & \multicolumn{1}{c|}{ Organization Structure } \\
\hline$G c$ & $O(e+(m i+n j) \log (m i+n j)+U(1))$ & $\begin{array}{l}\text { Fully Centralized, optimized by } \\
\text { minimum tree }\end{array}$ \\
\hline$G m$ & $O(m 2 n)$ & $\begin{array}{l}\text { Multiple centralized, unified stand- } \\
\text { ard rule }\end{array}$ \\
\hline$G n$ & $O(2 m+n)$ & $\begin{array}{l}\text { Multiple centralized, no unified } \\
\text { standard rule }\end{array}$ \\
\hline$G p$ & $O(2 m+i p+n+j p)$ & $\begin{array}{l}\text { Partially centralized, with some } \\
\text { unified arrangements }\end{array}$ \\
\hline$G l$ & $O(2 m+n+i+j)$ & Decentralized autonomous agents \\
\hline
\end{tabular}

$\mathrm{m}, \mathrm{n}$ : number of PP, TPP; i, j: number of the APIs they provide; number of the connecting links.

Fig. 8 Complexity of Ecosystems

A well-designed intermediate mechanism will reduce the complexity of the ecosystem. According the analysis of federation game, we should avoid to let the ecosystem controlled by dominated participants because the superiority will monopolize the resources and incomes. The complexity also concerns the API design. As stated above, the ecosystem can only control the partner API of the open zone and the effective mechanisms of the DMZ. If this is not well-done, the efficiency of the econsystem will be low.

When it is not well designed, even a small change will cause consequently influence of expotential degree. In the worst condition, $\boldsymbol{G} \boldsymbol{l}$ of mode 5 will be millions times higher than that of the others. And, this happens not only once but it needs such more amount of computing power 
Optimization of ICT Common Wealth Planning and Sharing based on Organic Economic Ecology and Theory of Knowledge Value Transformation

Johannes K. Chiang

when each time a small change occurs. In other words, a bad design will continuously consume the energy of the ecosystem and waste great amount of resources. More larger the scale of the ecosystem, more important a centralized deployment is. And, the more the number of centralized institutions, the higher the efficiency of the network.

\subsection{Analysis of the aviaiblity and value wrt. the rules for optimization}

The rules used in different organization structures imply the law or consensus for the optimization according to the Theory of Knowledge Value Transformation. Since mode 1 can only use minimum tree, the complexity is the highest. The less of the specific internal rules, the higher the efficiency of the ecosystem. It means open API is better than private API. When the number of the specific rules is small, the cycle time will reduce in a significant manner. In other words, the performance of the ecosystem becomes better when the rules are more standardized, i.e. open.

Even if there exists special rules, a centralized entity can use the compatible general purpose standard but the administration task will become the cost of the network when such kind of organization does not exisit. More possibily, the efficiency becomes worser and the users will suffer the costs, i.e. less of value, because there is no concensus.

A such kind of centralized organization slows down the speed of establishment of the open ecosystem. However, once such kind of oranization is established, the owner of the existing specific internal rules should modify their rules in order to follow up the consistency of the overal system. Form the systematicn viewpoint, the relatively large institutions should participate the open ecosysem beause they have richer resources and information that can be open for common welth.

Results of Analysis of Complexity i.e. efficiency, risk regarding security and costs, can be summarized as following:

- Fully centralized regime can accomplish the development and update of the ecosystem within polynomial time complexity.

- Partially centralized group with some unified arrangements is worse than Multiple Centralized Organic agents. With consideration of the communication costs in practice, fully centralized regime is even worse than fully autonomous agents.

- The costs for maintenance and participating the ecosystem could be the extra social cost and inevitably the friction costs of consequent resource exchanges.

- By the fully centralized regime, only the dominant agent can benefit from the ecosystem because the governance lies in the system and the influence will be greater in corresponding to the expansion of networking. For the situations of deadlock of policy or difficulty of building the law or consensus, it is the possible way to enter open systems.

As the result, "Open" is the best Consensus for the optimization. The federated cloud with unified rules for Open APIs is most efficient and effective as well as less of costs, more robust for security, less of risks. Thus, open collaboration framework based on federated cloud becomes the next edge of the planning and sharing ICT resources in the API and Platform Economy.

\section{Conclusion}


Optimization of ICT Common Wealth Planning and Sharing based on Organic Economic Ecology and Theory of Knowledge Value Transformation

This paper introduces in the first place the HyQVIS organic ecology, the Theory of Knowledge Value Transformation and the cooperative competition strategy for improvement. For the value aspect, pragmatism and altruism are taken into account to achieve the inclusive economy.

Since HyQVIS emphazes efficiency, minimum risk, rational distribution, this research applies Pareto Optimality as the optimization method for planning and sharing the resouces and further develops Pareto Frontier to improve the effecieny. In order to realize Pareto Frontier with scientific base, this research applies ISO 25000 to define the metrologic metrics for the fatures derievd from Pareto Frontier. For the realistic measure of value, this research carries out the values with respect to planner, proviieder and users of the services. Bsed on this theoritical baseline, this research conducts a case study of open collaboration framework for federated cloud with respet to API and Platform Economy.

According to HyQVIS, planning and sharing of the resouces is in the fact a cooperative game. This research appplies the Shapley value with consideration of inclusive economy. For this purpose, comprhensive factors influencing the organization of organic ecosystem are considered, including feasible state stable core, cooperation, hidden conflicts of self-regulation API Security etc. On top of these consideration, different organization structures of the ecosystems are constructed. The complexity, including efficiency and risk, aviaiblity and value are analysed. The result shows, "open" is the best consensus for optimiization of the common wealth, and the federated cloud is most efficient as well as less of complexity and costs, more robust for security, less of risks. Federated cloud becomes the next edge of the infrastructure of collaboration framework in the API and Platform Economy.

Blockchain is a kind of decentralized and distributed system where every node/VM owns the blockchain of data on their local storage. On the contrary, Cloud is a kind of centralized system and saves the data on the network. It is difficult to joint Blockchain and Clouds. Using API aided Federated Computing to connect Blockchain and Cloud could be the best way to solve the problem.

The contribution of this research can be summarized as follwoing:

1. Optimizing ICT Common Wealth Planning and Sharing with the Organic Ecology and Theory of Knowledge Value Transformation.

2. Optimizing the open ecosystem with the consideration of Altruism and Inclusive Economy.

3. Applying Pareto Optimality form Economics as the optimization method for planning and sharing the resources and further using Pareto Frontier to improve the efficiency.

4. Applying ISO 25000 to build the scientific metrological foundation for the metrics for the features derived from Pareto Frontier.

5. Extending of the value metrics of HyQVIS for API and Platform Economy.

6. Applying Shapley value as the game theory to enhance the cooperative competition strategy of HyQVIS to design the open collaboration Framework and ensure equilibrium of the ecosystem.

7. Utilizing the federation game of Major Service Providers and TPP to enlarge the incomes.

8. Explaining the characteristics of Open API and its security as well as efficiency, and analyzing the complexity of different organization structures.

9. Recommendation for open ICT ecosystems according to the result of the analysis. 
Optimization of ICT Common Wealth Planning and Sharing based on Organic Economic Ecology and Theory of Knowledge Value Transformation

10. Combining the theoretical concepts and practical experiences to develop the cloud infrastructure for open collaboration framework.

\section{References}

[1] J. K. Chiang, Qualitatsplanungssystematik auf der Basis von Hypertext-Techniken -- Ein Beitrag zur rechnerunterstutzten Teamarbeit in der ganzheitlichen Qualitatsplanung, Verlag Mainz, Wissenschaftsverlag, Aachen Germany1994, ISBN:3-930085-90-93.

[2] Wikipedia, Synergetics (Haken), Access time: Jan. 2020.

[3] Wikipedia, Yin-Yang and Five Elements, Acces tiime: Dec, 2020.

[4] J. K. Chiang, The new Hightech Service Industry and Regional Economy, Report and Special Issue to "2006 HighTec and Venture of North Taiwan" Project, Taipei City Government, ROC, Dec. 2006.

[5] J. K. Chiang, et al, Confucian Entrepreneurs and Far-East Culture, Journal of Zhejiang University (Humanities and Social Sciences), Vol.31 No. 1, pp. 5-15, Jan. 2007

[6] Wikipedia, Ecology, https://en.wikipedia.org/wiki/Ecology, Acces tiime: Dec, 2020.

[7] A. J. Toynbee, A Study of History, Oxford University Press, 1934-1961.

[8] Wikipedia, Information Theory, https://en.wikipedia.org/wiki/Information theory, Acces tiime: Dec, 2020.

[9] A Factory-wide Quality Information System based on Hypertext, HYQIS EU FP3-ESPRIT 3 ID: 8733, 1993, https://cor dis.europa.eu/project/id/8733.

[10] J. K. Chiang, Strategy, Knowledgy and Ecology for Strategical Service Industry, Report to EComerce, NICI ROC, Dec. 2014.

[11] Johannes K. Chiang, Kuang-Li R. Hung, Exploring Effects of Free Trail to the Diffusion Value of Mobile Application, BAI 2013 International Conference on Business and Information, Bali, Indonesia, 07-09 July 2013.

[12] Pareto Optimality, https://en.wikipedia.org/wiki/Pareto_efficiency.

[13] ISO 25000, https://iso25000.com/index.php/en/iso-25000-standards

[14] Johannes K. Chiang, Yi-Fang Chiang and Eric Yen, Optimization Of Spectrum Auction in 5th Generation Mobile Networks By The Optimization Algorithm The Simulated Annealing Algorithm And Genetic Algorithm For The Spectrum Below 6 GHz, ICCT/IEEE 2018, Aug. 8-10, 2018.

[15] Johannes K. Chiang, Ada Hui-Chuan Chen, Gestalt of Acceptance Model for Commercialized Technology Validated on the Case of Internet Banking from the Perspective of Users' Trust and Willingness, The 8th Asia Pacific Industrial Engineering and Management Systems and 2007 Chinese Institute of Industrial Engineering, Dec. 2007.

[16] J. Moilanen, M. Ninioja, M. Seppanen and M. Honkanen, API and Economy 101, Bod - Books on Demand, Helsinki, Finland, 2019.

[17] Eric Yen and Johannes Chiang, Development of Open Collaboration Framework for Disaster Mitigation, in: Advances and New Trends in Environmental Informatics Managing Disruption, Big Data and Open Science, Springer, Dec. 2018.

[18] Wikipedia, Federation (information technology), https://en.wikipedia.org/wiki/Federation_(information technology)

[19] Wikipedia, Shapley value, https://en.wikipedia.org/wiki/Shapley_value 